\title{
Investigating the nucleus-nucleus potential at very short distances
}

\author{
C. H. Dasso ${ }^{1}$ and G. Pollarolo ${ }^{2}$ \\ ${ }^{1}$ Department of Atomic, Molecular and Nuclear Physics, University of Sevilla, Apartado 1065, 41080 Sevilla, Spain \\ ${ }^{2}$ Dipartimento di Fisica Teorica, Universitá di Torino, and Istituto Nazionale di Fisica Nucleare, Sezione di Torino,
}

Via Pietro Giuria 1, 10125 Torino, Italy

(Received 27 March 2003; published 17 November 2003)

\begin{abstract}
Recent progress in experimental techniques have made possible accurate measurements of fusion cross sections far below the Coulomb barrier, revealing an unexpected behavior of these quantities as a function of the bombarding energy. Besides providing a plausible cause for the observed energy dependence we profit from the nature of this explanation and the high sensitivity of the experimental data to show how one can use these measurements to investigate the radial dependence of the nuclear ion-ion potential at extremely close distances.
\end{abstract}

DOI: 10.1103/PhysRevC.68.054604

In heavy-ion peripheral collisions close or above the nominal Coulomb barrier, the results of microscopic calculations of reaction cross sections for quasielastic processes are mostly sensitive to the value of the nucleus-nucleus potential and inelastic/transfer form factors at distances well outside the touching radius between the projectile and target. While the value of the Coulomb barrier is safely estimated [1] (although not better than to a level of a few percent) there is considerable ambiguity with regards to the actual radial dependence of the nuclear part of the ion-ion potentials that can be made consistent with the experimental findings. It is worth mentioning here that theoretical developments are able, within the frame of their assumptions, to predict the shape of the potential for distances well inside of the contact radius [2-4].

In this context, measurements of sub-barrier fusion cross sections - a field that continues to attract much experimental and theoretical attention [5-7] — became one of the most effective tools to shed light on the characteristics of the nuclear potential at shorter distances. In fact, the standard approach to investigate this class of phenomena exploits the mechanism of barrier penetration in the presence of couplings to intrinsic degrees of freedom [8-12]. Crucial to the success of these analyses is a proper adjustment of the height and thickness of the potential barrier for small partial waves $(\ell \approx 0)$. While the former controls displacements in the energy scale, the barrier width is related (for fusion dominated by negative $Q$-value channels, at least) to the exponential slope $2 \pi / \hbar \omega_{B}$ of the function $\sigma(E)$ at the lowest energies.

Sub-barrier fusion cross sections are essentially determined by only these two parameters in a small range of energies lower than but close to the barrier. To be precise, in the range of validity of the parabolic approximation when the effective potential is expressed in the neighborhood of its maximum, namely,

$$
V(r) \approx V_{B}-\frac{\mu \omega_{B}^{2}}{2}\left(r-r_{B}\right)^{2}
$$

where $r_{B}$ indicates the location of the barrier and $\mu$ the reduced mass of the system. (Actually, the parameters $V_{B}$, $r_{B}$, and $\hbar \omega_{B}$ should be all labeled by the partial wave
PACS number(s): 25.70.Hi, 21.30.Fe, 24.10.-i, 25.70.Jj

number $\ell$.) The severe exponential drop associated with the characteristic values of $\hbar \omega_{B}$ has limited in practice the range of measured cross section to a span of energies of only a few $\mathrm{MeV}$, a small percent change of the bombarding energies with respect to the barrier value $V_{B}$. In such case the parabolic approximation remains valid.

A pioneering experiment in Argonne has gone well beyond this limit prompting the experimental group to report their results as "unexpected" in a recent paper [13]. However, a large number of ion-ion potentials predict a pocket in the inner region, just as is illustrated in Fig. 1. Here we see-for the particular reaction covered in Ref. [13] - the profile of a frequently used ion-ion potential (the AkyüzWinther potential of Ref. [4]). Shown in the figure is also the absolute limit to fusion that comes from the required exothermic character of the process that was mentioned by the authors of Ref. [13]. This energy is far below the relative one quenching the tunneling process, $V_{P}$, and therefore it is clear that the latter takes precedence as a limiting mechanism causing $\ln \sigma(E) \rightarrow-\infty$. Furthermore, since somewhere half the way between the maximum and the minimum there should be an inflection point, one can safely conclude that deviations from the parabolic approximation should be expected to show up even much earlier as the bombarding energy is gradually reduced below the barrier. A simple, visual estimation of the value of energy where this would happen is shown by the arrow, less than $5 \mathrm{MeV}$ below the barrier. This number is very much consistent with the findings of Ref. [13].

It can be appreciated in Fig. 1 that the largest departure from the parabolic behavior is associated with the slow radial dependence of the monopole Coulomb interaction term for distances larger than $r_{B}$. However, this component of the potential has a well-defined shape, does not introduce any ambiguity in the transmission coefficients, and therefore it is quite under control.

Our contention in this paper is that, on the other hand, it is possible to turn this situation to our advantage and use the outstanding quality of the data of the experiment performed in Argonne (or similar) to learn about the shape of the inner side of the potential barrier. Or, equivalently, to gain empirical knowledge of the nucleus-nucleus potential at much 


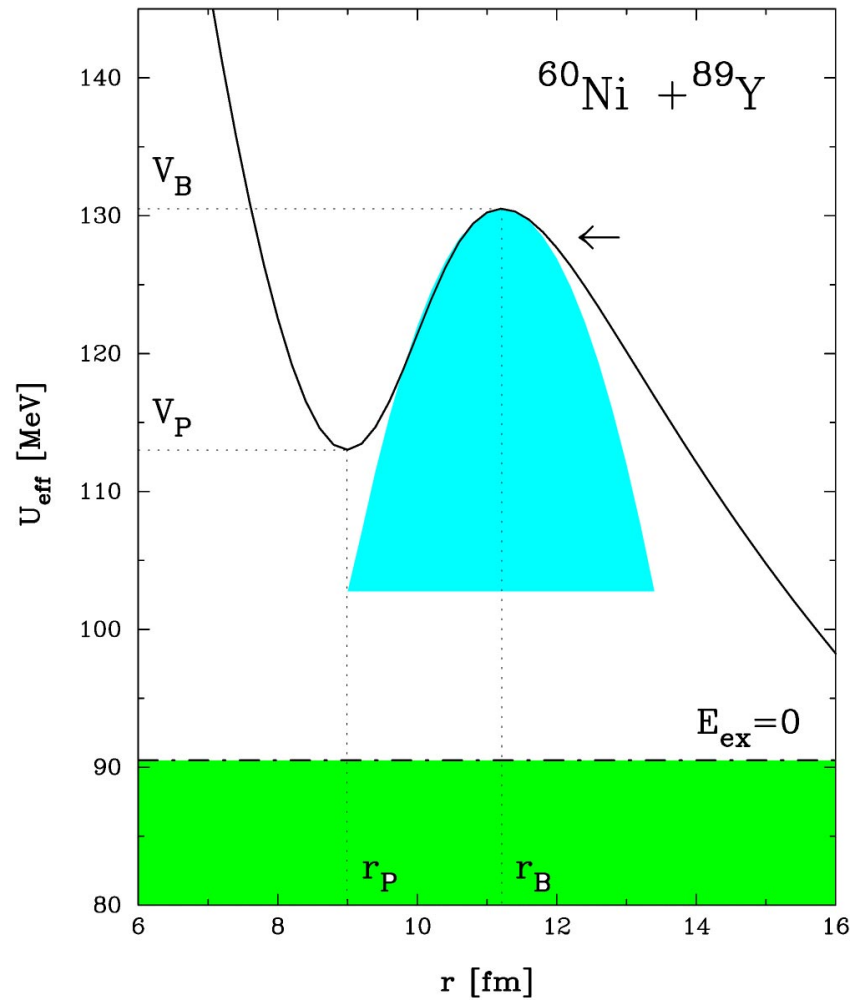

FIG. 1. (Color online) The nucleus-nucleus potential for a head-on collision $(\ell=0)$ in the reaction ${ }^{60} \mathrm{Ni}+{ }^{89} \mathrm{Y}$. The nuclear part of the interaction is given by the Akyüz-Winther potential. Shown in the figure is also the profile of the parabolic approximation that results from approximating the potential near the top of the barrier, $V_{B}$, by a quadratic dependence in $r$. The inner pocket $V_{P}$ is in this case about $15 \mathrm{MeV}$ below the barrier, at an energy considerably larger than the value of $\approx 90 \mathrm{MeV}$ that sets the absolute lower limit for which the fusion process can energetically occur.

shorter distances than hitherto achieved.

To this end we shall show that there is enough sensitivity in the deviations from the pure exponential decay of the cross sections for very low energies (where they occur, by how much, etc.) to discern between different potential profiles in that inner region. We will limit ourselves to show the way in which these considerations influence the fusion cross sections in the absence of interactions with internal degrees of freedom. How the couplings to different reaction channels affect the so-called "reference" curve has been extensively covered in the literature [8-12] and we know that the final results at the lowest bombarding energies always inherit (or build upon) whatever characteristics are already present in the simplest barrier-penetration formulation of the problem.

Clearly, we cannot rely any longer in the analytic form of the transmission coefficients for a parabolic barrier and we shall use, in what follows, their Wentzel-Kramers-Brillouin expression [4]. The implementation of this prescription requires a numerical integration for each value of the bombarding energy and each effective potential, as defined by the partial wave number $\ell$. The procedure, however, cannot be used for all values of $E<V_{B}$ and needs to be complemented by the analytic expressions which are valid near the top of the barrier. Fortunately, there is a wide range of energies
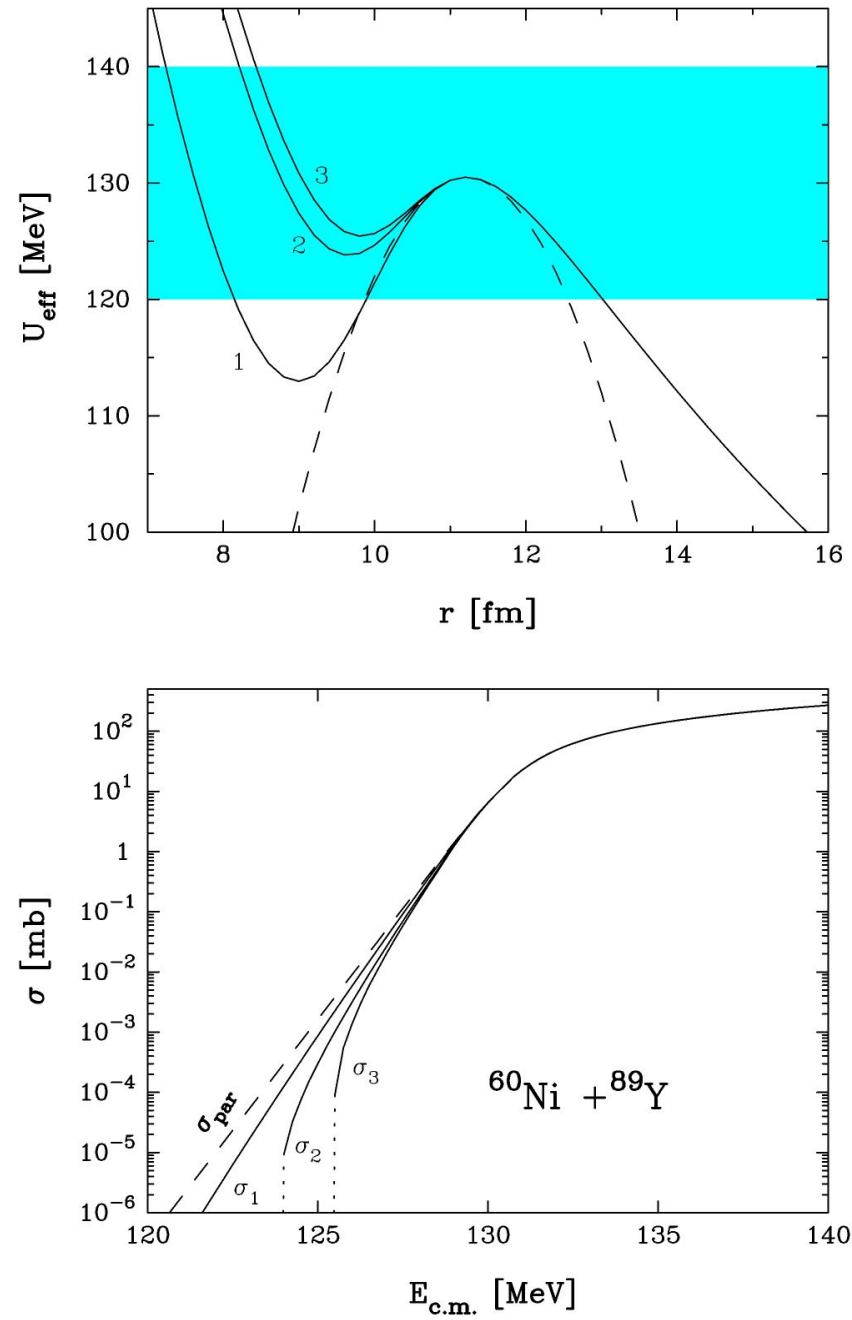

FIG. 2. (Color online) (top) Three possible ion-ion potentials for the reaction ${ }^{60} \mathrm{Ni}+{ }^{89} \mathrm{Y}$ having an identical radial dependence for $r>11.01 x \mathrm{fm}$. This is a matching distance chosen somewhat inside the barrier radius and, consequently, the values of $V_{B}, r_{B}$, and $\hbar \omega_{B}$ are shared by all three potential functions. The matching is done with the purpose of generating different alternatives for the inner side of the potential barrier. The curves shown are for the partial wave $\ell=0$ but a similar prescription can also be implemented for the effective potentials corresponding to all the other low values of $\ell$ which are relevant for a calculation of sub-barrier fusion cross sections. Also shown in the frame is the parabolic approximation that is common to the three potentials. (Bottom) The fusion cross sections as a function of bombarding energy in the center of mass for the four potential profiles shown above. $\sigma_{\text {par }}(E)$ is the cross section within the parabolic approximation while $\sigma_{1,2,3}(E)$ correspond to each of the potential functions as identified in the upper frame.

where the results of either alternative coincide and thus a smooth matching of the transmission functions poses no technical difficulty.

Our strategy consists in taking a family of hypothetical potentials that are identical for large values of $r$ and which share the same values of $V_{B}, r_{B}$, and $\hbar \omega_{B}$. The only difference between these potentials occurs for distances somewhat inside of the barrier position where they are matched in value 
and first derivative to parametrized functions that are thus able to recreate various radial dependences for the left-hand side of the potential barrier.

An example of three such potentials is shown in the top frame of Fig. 2. The reaction is again ${ }^{60} \mathrm{Ni}+{ }^{89} \mathrm{Y}$ and the matching has been done at $r=r_{B}-0.2 \mathrm{fm}$, what makes all three ion-ion interactions identical for $r>11 \mathrm{fm}$. For the sake of comparison we also show in the same frame the parabolic potential approximation that is, by definition, common to all three functions. Within this approximation all the potentials lead to the same fusion reaction cross section as a function of the bombarding energy, $\sigma_{p a r}(E)$, shown with a dashed line in the bottom frame of Fig. 2. This function reflects the behavior that is characteristic of this approximation; an exponential falloff towards the low energies with a slope entirely controlled by the curvature of the barrier at its top.

Cross sections are displayed in Fig. 2 in the interval $120 \mathrm{MeV}<E_{\text {c.m. }}<140 \mathrm{MeV}$, which corresponds to the shaded range of energies indicated in the upper frame, where c.m. stands for center of mass. In this energy interval one should be able to observe the total quenching of the fusion cross sections corresponding to the potentials 2 and 3 , since the values of $V_{P}$ for these potentials fall within the selected energy range. This is indeed the case as it can be seen in the lower frame, where the curves have been completed with a dotted vertical line to guide the eye to the values of the abscissa where $\ln \sigma_{2,3} \rightarrow-\infty$. The drastic cutoff occurs, in these examples, for cross sections in the range of microbarns to nanobarns. Small as they may seem, we recall that the orders of magnitude of these quantities do not differ much from the cross-section values reported in the Argonne experiment.

It is important to note that the quantity $\Delta^{\ell}=V_{B}^{\ell}-V_{P}^{\ell}$ acquires its maximum value for $\ell=0$. In fact, as the centrifugal contribution to the effective potential increases, the relative depth of the pocket reduces (until it completely disappears at a critical value $\ell_{c r}$ ). This explains why, in the accumulation of partial-wave contributions, which builds up the fusion cross section, the effects we are discussing end up being perhaps more pronounced than one could have expected. To start, phase-space considerations favor the contribution of the larger partial waves. But, in addition, deviations from the parabolic behavior set in at an earlier stage, i.e., at a smaller number of MeV's below the corresponding barrier height.

The nature of this explanation is quite general and does not rely on the characteristics of any intrinsic channels that may be active in a particular reaction. Thus, the elements we have discussed should be present, in a more or less prominent way, in many other reactions besides the specific one we have chosen for illustration. This is in agreement with the analysis of Ref. [13], where plenty of evidence is provided to this effect.

It is possible, with this approach, to learn about the inner shape of the nucleus-nucleus interaction because of the sharp, exponential dependence of the tunneling probabilities with respect to details of the potential in the zone where classical motion is forbidden. This extraordinary sensitivity is what causes the range of lifetimes for $\alpha$ decay to span forty orders of magnitude (and what, incidentally, provided one of the earliest procedures for the estimation of nuclear radii). In a sense, the difficulties posed by the precise determination of lifetimes exceeding millions of years are a counterpart to those of measuring cross sections of the order of nanobarns in the context of our present problem. The feasibility of achieving such goal was, however, proved by the work of Ref. [13]. It is important to encourage the continuation of these efforts and the accumulation of further data in order to reveal, as unambiguously as possible, the characteristics of the ion-ion interaction at extremely short distances.
[1] L. C. Vaz, J. M. Alexander, and G. R. Satchler, Phys. Rep. 69, 373 (1981).

[2] J. Blocki, J. Randrup, W. J. Swiatecki, and C. F. Tsang, Ann. Phys. (N.Y.) 105, 427 (1977).

[3] Ö. Akyüz and A. Winther, Proceedings of the International School of Physics "Enrico Fermi," Course LXXVII, edited by R. A. Broglia, C. H. Dasso, and R. Ricci (North-Holland, Amsterdam, 1981).

[4] R. Broglia and A. Winther, Heavy Ion Reactions (AddisonWesley, Redwood City, CA, 1991).

[5] A. B. Balantekin and S. Kuyucak, J. Phys. G 23, 1159 (1997).

[6] M. Dasgupta, D. J. Hinde, N. Rowley, and A. M. Stefanini, Annu. Rev. Nucl. Part. Sci. 48, 401 (1998).
[7] G. Pollarolo and A. Winther, Phys. Rev. C 62, 054611 (2000).

[8] H. Esbensen, Nucl. Phys. A352, 147 (1981).

[9] C. H. Dasso, S. Landowne, and A. Winther, Nucl. Phys. A405, 381 (1983).

[10] C. H. Dasso and S. Landowne, Comput. Phys. Commun. 46, 187 (1987).

[11] H. Esbensen and S. Landowne, Phys. Rev. C 35, 2090 (1987).

[12] K. Hagino, N. Rowley, and A. T. Kruppa, Comput. Phys. Commun. 123, 143 (1999).

[13] C. L. Jiang, H. Esbensen, K. E. Rehm, B. B. Back, R. V. F. Janssens, J. A. Caggiano, P. Collon, J. Greene, A. M. Heinz, D. J. Henderson, I. Nishinaka, T. O. Pennington, and D. Seweryniak, Phys. Rev. Lett. 89, 052701 (2002). 